\title{
New role of E3 ubiquitin ligase in the regulation of necroptosis
}

\author{
Jinho Seo, Eun-Woo Lee EJ Jaewhan Song ${ }^{*}$ \\ Department of Biochemistry, College of Life Science and Biotechnology, Yonsei University, Seoul 03722, Korea
}

\begin{abstract}
Necroptosis is a well-known form of caspase-independent cell death. Necroptosis can be triggered by various extrinsic stimuli, including death ligands in the presence of receptorinteracting protein kinase 3 (RIPK3), a key mediator of necroptosis induction. Our recent studies have revealed that C-terminus HSC-70 interacting protein (CHIP), an E3 ligase, can function as an inhibitor of necroptosis. $\mathrm{CHIP}^{-1-}$ mouse embryonic fibroblast showed higher sensitivity to necrotic stimuli than wild-type mouse embryonic fibroblast cells. Deleterious effects of CHIP knockout MEFs were retrieved by RIPK3 depletion. We found that CHIP negatively regulated RIPK3 and RIPK1 by ubiquitylation- and lysosome- dependent degradation. In addition, CHIP $^{-1-}$ mice showed postnatal lethality with intestinal defects that could be rescued by crossing with RIPK3 ${ }^{-1-}$ mice. These results suggest that CHIP is a negative regulator of RIPK1 and RIPK3, thus inhibiting necroptosis. [BMB Reports 2016; 49(5): 247-248]
\end{abstract}

The cell death pathway can be divided into caspase-dependent and caspase-independent cell death pathways. Necroptosis, a form of programmed necrosis, is an extensively studied caspase-independent cell death pathway. It is considered as an alternative form of cell death pathway developed in the host to fight against viruses and bacteria. Necroptosis is triggered by the activation of death receptors, including tumor necrosis

*Corresponding author. E-mail: jso678@yonsei.ac.kr

http://dx.doi.org/10.5483/BMBRep.2016.49.5.067

Received 14 April 2016

Keywords: CHIP, Lysosome, Necroptosis, RIPK3, Ubiquitylation

Abbreviations: RIPK3, Receptor-interacting protein kinase 3; RIPK1, Receptor-interacting protein kinase 1; CHIP, C-terminus HSC70-interacting protein; TNF, Tumor necrosis factor; TLR, Toll like receptor; INFR, Interferone receptor; MLKL, Mixed lineage kinase domain-like; DAMP, Damage associated molecular patterns; HMGB1, High-mobility group box 1; PTEN, Phosphatase and tensin homolog; MEF, Mouse embryonic fibroblast; FADD, Fas-associated death domain

Perspective to: Jinho Seo et al (2016), CHIP controls necroptosis through ubiquitylation- and lysosome-dependent degradation of RIPK3, Nat Cell Biol. Mar 18(3) 291-302, doi:10.1038/ncb3314 factor (TNF) receptor, Toll-like receptors (TLR), and interferon receptors (IFNRs). Once activated, death receptors recruit a variety of signaling molecules to form primary complexes such as TNF complex $\mathrm{I} / \mathrm{Il}$ or death-inducing signaling complex. When caspase-8 activity is low or inhibited, necrosome complexes are formed. Necrosomes are composed of phosphorylated receptor-interacting protein kinase 1 (RIPK1) and RIPK3. Activated RIPK3 induces the phosphorylation of mixed lineage kinase domain-like protein (MLKL) which leads to $M L K L$ oligomerization. Oligomerized MLKL moves to the plasma membrane and disrupts the membrane integrity directly or indirectly. While MLKL might be the final effector molecule of necroptosis, other unknown factors affecting these final steps cannot be excluded. In contrast to apoptosis, necroptosis induces the release of damage-associated molecular patterns (DAMP) such as high-mobility group box 1 (HGMB1) that can lead to inflammation by activating innate immune cells. Recent studies have shown that several disease models related to apoptosis such as ischemia-reperfusion injury and neurodegeneration are also associated with necroptosis. While more detailed in vivo studies are necessary, these observations indicate that necroptosis involves functionally integrated pathways and causes a variety of human diseases.

Our study revealed that C-terminus HSC70-interacting protein (CHIP) could function as a negative regulator of necroptosis. CHIP is known as an UBOX-containing E3 ligase with tetratricopeptide as a chaperone-binding motif. CHIP regulates various tumor suppressor proteins such as p53, phosphatase and tensin homolog (PTEN), thus protecting cells from apoptosis. However, the function of CHIP in extrinsic or non-apoptotic cell death signaling remains unclear. Since hyperactivation of necroptosis is linked to the disruption of tissues or lethality, we evaluated whether non-apoptotic cell death was activated in $\mathrm{CHIP}^{-1-}$ mice. To test this, we first employed CHIP knockout mouse embryonic fibroblasts (MEFs) to study necroptosis.

Based on our results, CHIP $P^{-1-}$ MEFs showed higher sensitivity to necroptosis and promoted necrosome complex formation in response to necrotic stimuli. Western blotting data revealed that $\mathrm{CHIP}^{-1-}$ MEFs had higher RIPK1 and RIPK3 protein levels and stabilities compared to wild-type MEFs. We also showed that CHIP could degrade RIPK1 and RIPK3. In addition, CHIP directly ubiquitylated RIPK1 and RIPK3 under exogenous and endogenous conditions. Finally, CHIP-mediated

ISSN: 1976-670X (electronic edition)

Copyright (c) 2016 by the The Korean Society for Biochemistry and Molecular Biology

(c) This is an open-access article distributed under the terms of the Creative Commons Attribution Non-Commercial License (http://creativecommons.org/licenses/by-nc/4.0) which permits unrestricted non-commercial use, distribution, and reproduction in any medium, provided the original work is properly cited. 


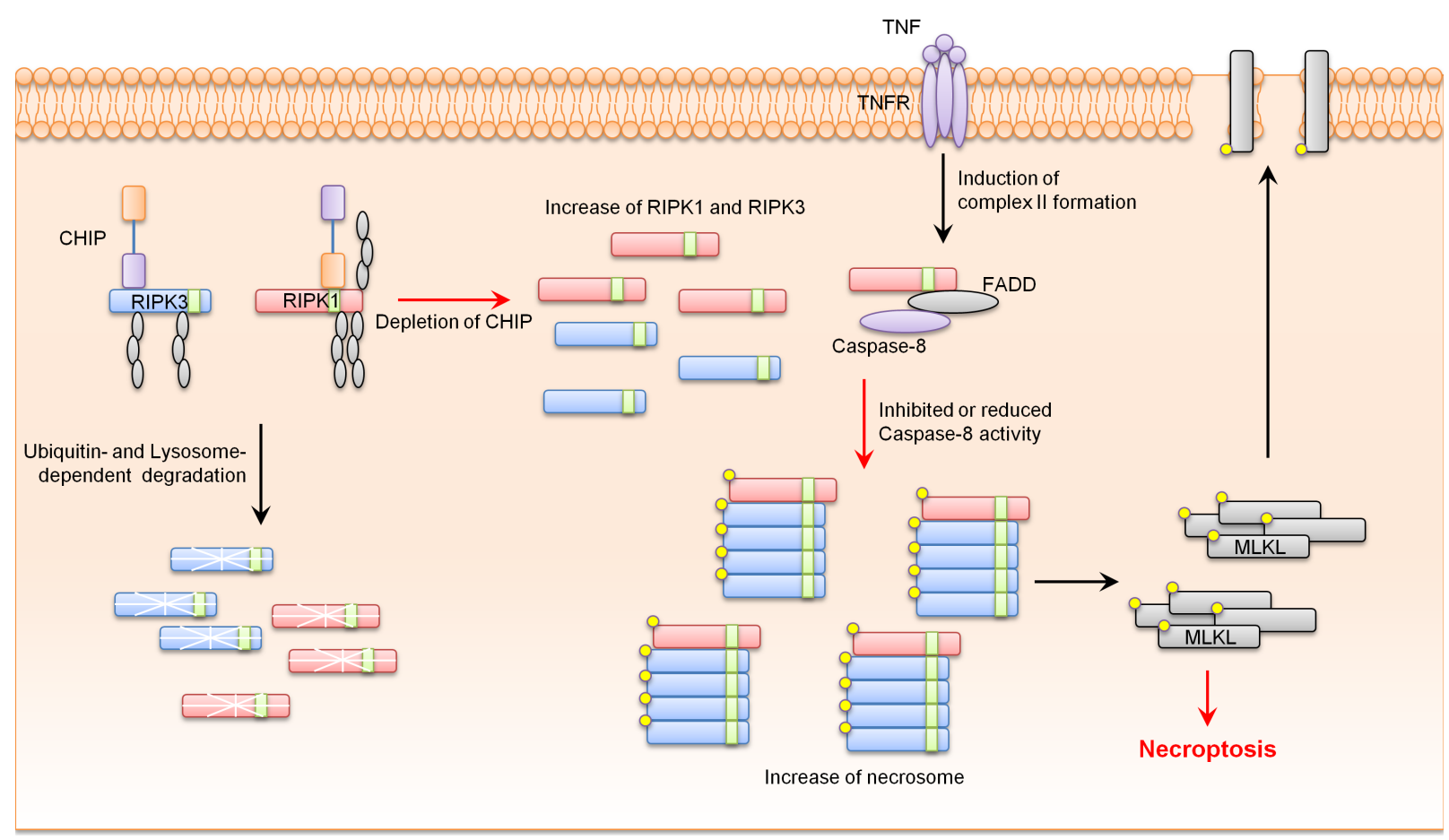

Fig. 1. CHIP inhibits necroptosis by ubiquitylation- and lysosome- dependent RIPK1 and RIPK3 degradation.

ubiquitylation of RIPK1 and RIPK3 occurred at lysine 571, 604 , and 627 of RIPK1 and lysine 55 and 363 of RIPK3, leading to lysosomal localization of RIPK1 and RIPK3 (Fig. 1).

Recently, intestinal epithelial cell-specific knockout of Fas-associated death domain (FADD) or caspase-8 has been shown to be able to induce massive cell death and inflammatory phenotypes in mouse intestinal epithelial cells. These phenotypes were rescued by treatment with necrostatin-1, a necroptosis inhibitor, or crossing with RIPK3 knockout mice, indicating the critical role of necroptosis in intestinal defects. Our analysis showed that $\mathrm{CHIP}^{-1-}$ mice died within 4-8 weeks with a high rate of cell death in the intestinal epithelium, a typical phenotype of necroptotic animal models. These phenotypes of $\mathrm{CHIP}^{-1-}$ mice were rescued by crossing with $R I P K 3^{-1-}$ mice. There was no change in cleaved-caspase- 3 protein levels in the intestines of $\mathrm{CHIP}^{-1-}$ mice, indicating that intestinal defects in $\mathrm{CHIP}^{-1-}$ mice occurred in an apoptosis-independent manner. RIPK1 and RIPK3 protein levels were increased in the intestines of CHIP ${ }^{-1-}$ mice, suggesting that CHIP deficiency could lead to the activation of necroptosis by up-regulating the levels of RIPK1 and RIPK3 proteins. Although further analyses of organs in $\mathrm{CHIP}^{-1-}$ mice related to necroptosis are required, our data suggest that CHIP can negatively regulate necroptosis in the intestinal epithelium by ubiquitylation-mediated and lysosome-dependent degradation of RIPK1 and RIPK3.

\section{ACKNOWLEDGEMENTS}

This work was supported by a grant (NRF-2015R1A3A2066581 to J. Song) from the National Research Foundation of Korea funded by the Ministry of Science, ICT and Future Planning, Republic of Korea. 